F. Child Lang. 34 (2007), I-20. (C) 2007 Cambridge University Press doi:I0.1017/So305000906007689 Printed in the United Kingdom

\title{
Reference and attitude in infant pointing*
}

\author{
ULF LISZKOWSKI, MALINDA CARPENTER \\ AND MICHAEL TOMASELLO \\ Max Planck Institute for Evolutionary Anthropology, Department of \\ Developmental and Comparative Psychology, Leipzig, Germany
}

(Received 24 March 2005. Revised 6 fuly 2006)

\begin{abstract}
We investigated two main components of infant declarative pointing, reference and attitude, in two experiments with a total of ro6 preverbal infants at $\mathrm{I} ; \mathrm{O}$. When an experimenter $(\mathrm{E})$ responded to the declarative pointing of these infants by attending to an incorrect referent (with positive attitude), infants repeated pointing within trials to redirect E's attention, showing an understanding of E's reference and active message repair. In contrast, when $\mathrm{E}$ identified infants' referent correctly but displayed a disinterested attitude, infants did not repeat pointing within trials and pointed overall in fewer trials, showing an understanding of E's unenthusiastic attitude about the referent. When $\mathrm{E}$ attended to infants' intended referent AND shared interest in it, infants were most satisfied, showing no message repair within trials and pointing overall in more trials. These results suggest that by twelve months of age infant declarative pointing is a full communicative act aimed at sharing with others both attention to a referent and a specific attitude about that referent.
\end{abstract}

\section{INTRODUCTION}

Infants sometimes point to objects for other people even when they do not want to obtain those objects. One reason they might do this is to direct others' attention to the objects so that they can then share attention and interest with others about them (so-called 'proto-declarative' pointing; Bates, Camaioni \& Volterra, I975; Camaioni, I993). However, Moore \& D'Entremont (200I) found that one-year-old infants pointed in this manner even if an adult was already attending to the object prior to the point. Therefore, they concluded that infants do not point to direct others'

[*] Thanks to Konstanze Albrecht for coding and helping with the data collection and to anonymous reviewers for helpful comments on a previous version. Address for correspondence: Ulf Liszkowski, Max Planck Institute for Evolutionary Anthropology, Deutscher Platz 6, D-o4ro3 Leipzig, Germany. e-mail: liszkowski@eva.mpg.de 
attention to objects (and do not even possess a social-cognitive understanding of others' attention). Instead, they proposed that infants point to obtain adult attention to the self, not to the object.

Liszkowski, Carpenter, Henning, Striano \& Tomasello (2004) challenged this latter account of infant declarative pointing both theoretically and empirically. They argued that infants do in fact understand something about others' attention but may sometimes point to something that another person is already looking at when they want to express their interest in it and share this interest with the other person. To test the competing accounts empirically, Liszkowski et al. (2004) had an experimenter (E) react in different ways to twelve-month-old infants' pointing at interesting events, with the question being how infants would react to these different adult reactions. To test Moore \& D'Entremont's (200I) hypothesis that infants only want to obtain attention to the self, E reacted to infants' points by attending only to the infant, not to the referent, and emoting positively to the infant (FACE condition). To test the complementary hypothesis that infants only wanted to direct attention to the referent, $\mathrm{E}$ reacted by attending only to the event, not to the infant, and not commenting or emoting about it (EvEnT condition). To test Liszkowski and colleagues' hypothesis that infants point to share attention and interest, E reacted by alternating attention to both referent and infant and commenting positively (JOINT ATTENTION condition). To test the hypothesis that infants do not intend to communicate at all, E reacted by ignoring infants' pointing and attending to neither the event nor the infant, not commenting on anything at all (IGNORE condition).

The main finding was that infants showed dissatisfaction in all but the Joint Attention condition. In all the other conditions, children pointed overall in significantly fewer trials, eventually giving up on pointing for E. Furthermore, when $\mathrm{E}$ did not alternate gaze between infant and event and comment positively about it, those infants who pointed repeated their pointing within trials significantly more often, presumably in an attempt to change E's response. Thus, infants were not satisfied when the adult attended either exclusively to themselves or to the object, but rather they wanted the adult to attend to both themselves and the object, so that the two of them could share attention and interest in the object. In line with Liszkowski et al. (2004) we therefore propose that infants' pointing in such a context has two components: ( $\mathrm{I}$ ) directing an adult's attention to some external event or entity in order to align the adult's attention with their own (shared attention); and (2) expressing interest or some other attitude to that shared event or entity in the expectation that the adult will align her own attitude with the infants' (shared attitude). Thus, even if the adult is already looking at an object, infants might still point to it in order to achieve their second goal of sharing an attitude about it. Like fully linguistic speech 
acts (Searle, I969; see also Bates et al., I975), infant declarative pointing thus embodies both reference and attitude.

In the current study, we investigated each of these two components in more detail. Following the basic design of Liszkowski et al. (2004), an adult reacted to infants' declarative pointing in various ways, and infants' reaction to that reaction was observed. With regard to reference, the adult either correctly identified the infants' intended referent or else alighted on some nearby, but incorrect, object as the infants' intended referent. With regard to attitude, the adult either expressed interest or disinterest in the object. There were thus four types of reactions resulting from a cross of the two factors of reference and attitude: (i) identify the referent correctly or incorrectly, crossed with (ii) share or not share the infants' attitude. Our hypothesis, which we will call the sharing hypothesis, was that infants would only be satisfied when the adult both correctly identified the referent (shared reference) and expressed the infant's attitude to that referent (shared attitude).

More specifically, with regard to reference, under the sharing hypothesis we predicted that infants would be satisfied only if the adult identified the correct referent. If the adult consistently misunderstood infants' referents and attended to something else, we expected that infants would be dissatisfied and thus would point on fewer trials across the session and/or would engage in some kind of message repair (Golinkoff, r 986) to correct the adult (i.e. would repeat pointing within trials to redirect the adult's attention to the correct referent). The competing hypothesis is that infants point instead to direct bodily behaviour and not attention (or perhaps, in a kind of triadic version of the hypothesis of Moore \& D'Entremont, 200 I, point simply to obtain adult attention or emotion about anythingirrespective of any particular referent). That hypothesis would predict that it would not be important for infants whether the adult referred to their precise referent or not, so when she did not, they would not engage in message repair by repeating pointing within trials. On that account, infants would be satisfied if the adult simply oriented and emoted appropriately toward something and thus they would point in as many trials across the session as when the adult correctly identified their referent.

With regard to attitude, under the sharing hypothesis we predicted that infants would be satisfied only if the adult shared their attitude of interest in the object. If the adult did not do this, we predicted that infants would eventually stop pointing across trials for the adult and would not engage in any kind of message repair. A competing hypothesis is that infants point simply in order to obtain positive emotions. This hypothesis would predict that infants would be dissatisfied when the adult emoted neutrally instead of positively and thus that they would persist in pursuing their goal in this case and would repeat pointing within trials - like they do when they try 
to obtain things by 'proto-imperative' pointing (Bates et al., I975). Alternatively, another competing hypothesis is that infants are requesting information in a kind of social referencing way: they point out an object or event for an adult and, in essence, inquire about the adult's attitude toward it or about its valence (see Baldwin \& Moses, I 996). In this case, any attitude the adult expressed, even neutral disinterest, would satisfy infants and they would thus point in as many trials across the session as when the adult emoted positively.

\section{MAIN EXPERIMENT}

In this experiment we tested the sharing hypothesis that E's reaction to infants' pointing needs to involve E's alignment to both their referent and their attitude about the referent in order to satisfy infants' communicative intent. We had three specific predictions. First, we predicted that in the three conditions in which $\mathrm{E}$ did not share both attention AND interest infants would point in fewer trials across the session, as they did in the study by Liszkowski et al. (2004), effectively giving up on pointing for that communicative partner. Second, we predicted that when E expressed interest but misunderstood the referent, infants would repeat pointing to the same referent within trials (in an attempt to redirect E's attention) more often than when $\mathrm{E}$ expressed interest about the correct referent. Third, we predicted that when $\mathrm{E}$ referred to the correct referent but did not share infants' positive attitude, infants would show no increase in pointing within trials because they were not simply 'ordering' $E$ to express positive emotions; following our first prediction, in this case they would point in fewer trials across the session, not being satisfied with this response.

\section{METHOD}

\section{Participants}

Eighty German infants were recruited from a database of infants whose parents had agreed to participate in studies of infant development. Only infants that were reported by their parents to be pointing were invited to participate. An additional 25 infants were excluded because of experimenter error $(n=5)$, fussiness $(n=\mathrm{I} 2)$, or lack of pointing $(n=8)$. Table I displays the number of included and excluded infants, mean age, range, and gender for each of four experimental groups $(n=20$ in each). Infants received a small gift for participating after the test.

\section{Materials and set-up}

Figure I shows a schematic drawing of the experimental set-up for two of the experimental conditions. Set-up and materials were mostly similar to 
INFANT POINTING

TABLE I. Main experiment: description of the sample

\begin{tabular}{|c|c|c|c|}
\hline & $\mathrm{N}$ (not included $\left.{ }^{\mathrm{i}}\right)$ & Age (range) & Male/Female \\
\hline Total & $80\left(\mathrm{I} 2^{\mathrm{a}} ; 5^{\mathrm{b}} ; 8^{\mathrm{c}}\right)$ & I;O.I5（O; I I.I6-I; I.I4) & $42 / 38$ \\
\hline Joint Attention & $20\left(0^{\mathrm{a}} ; \mathrm{I}^{\mathrm{b}}\right)$ & I; O.I6 (o; I I.I6-I ; I.I4) & $12 / 8$ \\
\hline Misunderstanding & $20\left(\mathrm{I}^{\mathrm{a}} ; 2^{\mathrm{b}}\right)$ & I;O.I3 (0; I I.20-I ; I.I 2$)$ & I0/10 \\
\hline Uninterested & $20\left(2^{\mathrm{a}} ; \mathrm{I}^{\mathrm{b}}\right)$ & I;O.I 4 (I ; O.IO-I ; I.07) & $12 / 8$ \\
\hline No Sharing & $20\left(3^{\mathrm{a}} ; 0^{\mathrm{b}}\right)$ & I ; O.I I (I ;O.IO-I ; I.II ) & $8 / 12$ \\
\hline
\end{tabular}

Notes: ${ }^{\mathrm{i}}$ Numbers in parentheses do not add up across conditions, because some infants did not point and could not be allocated to a condition. ${ }^{a}$ Additional infants tested but not included because of fussiness. ${ }^{b}$ Additional infants tested but not included because of experimenter error. ${ }^{\mathrm{c}}$ Additional infants tested but not included because of no points.

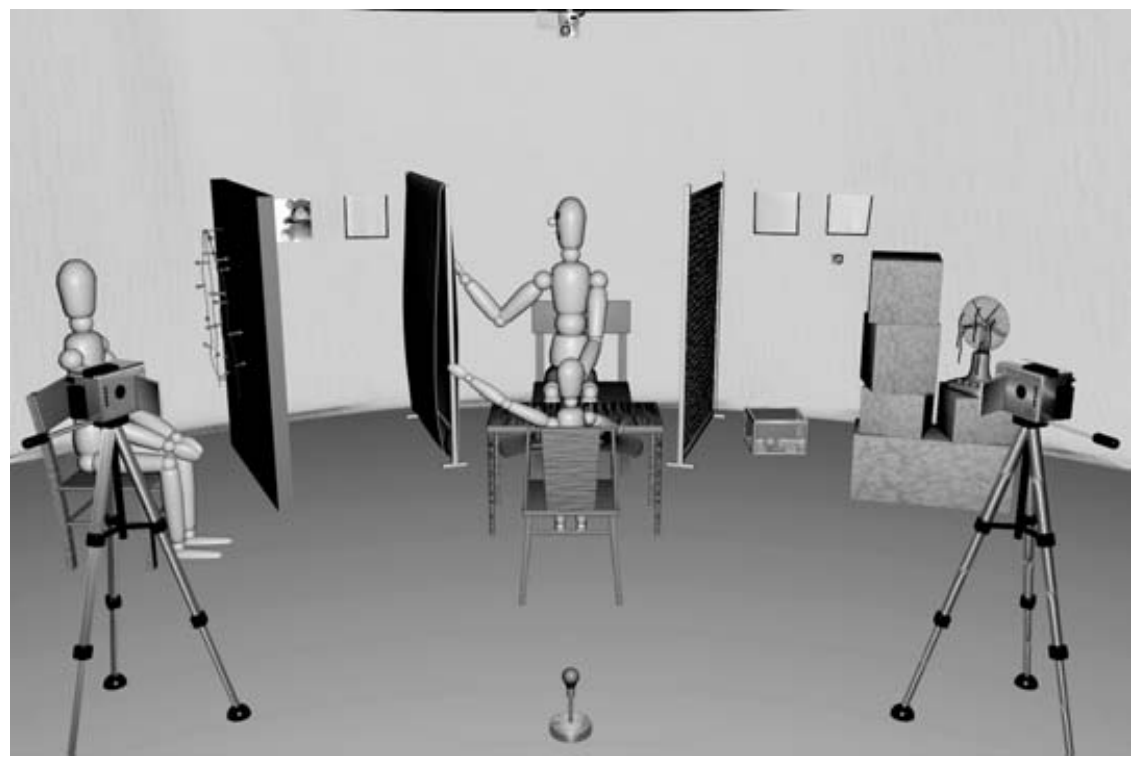

Fig. I. Schematic drawing of the set-up of the main experiment in the conditions involving barriers.

those used in Liszkowski et al. (2004). A semi-circular screen of white cloth sheets was positioned in front of the infant at a distance of $2.5 \mathrm{~m}$, blocking the entire back of the test room. The screen had four openings at a height of $90 \mathrm{~cm}$ from the floor, two at each side of the infant's midline at equal distances. These openings were covered with curtains which could be opened from behind. There were eight different stimuli : four hand puppets and four other objects. During each test trial a research assistant, E2, either protruded one of the hand puppets through one of the openings, or operated one of the four objects from behind the screen. These four objects were 
located at equal distances to the infant's midline. Three of them were always visible to infants in front of the screen and could be activated electronically (a string of white lights which flashed at I sec. intervals hanging on one of the barriers, a red light which flashed irregularly on the floor, and a fan with coloured stripes of crepe-paper attached on a stack of boxes). For the fourth object (a wooden bird with flapping wings) the opening on the infant's far left was reused. The order of the stimuli was the same for every infant.

E sat facing the infant, approximately $0.8 \mathrm{~m}$ away, with his back to the screen. Two of the experimental conditions (MISUNDERSTANDING and NO SHARING; see below) involved barriers which obstructed E's line of sight to the stimuli (see Figure I). Two of four barriers (a clothes rack and a wicker screen) stood proximal to E's left and right, each obstructing three stimuli locations distant to the barriers from E. The other two barriers (a solid cardboard wall and a stack of boxes) were more distal to $\mathrm{E}$ and each obstructed an object close to or attached to it from E. On the side of the barriers visible to $\mathrm{E}$ there were little papers attached or, hanging on the clothes rack, a jacket, all of which $E$ could reach. In the other two experimental conditions the set-up was identical but with no barriers present. The caregiver sat to the infant's left, slightly behind the infant.

One camera was positioned above the infant and one camera was positioned to the right of the infant's midline, both hidden behind cloth sheets, to record the infant. Another two cameras were positioned left and right of E's midline behind the infant, to record $\mathrm{E}$ and the stimuli. The cameras were connected to a quad-splitter that fed the image into a monitor behind the screen so E2 could monitor the infant's behaviour. A small light behind the infant was switched on by E2 to signal to E the onset and offset of each trial.

\section{Procedure}

All infants participated in a warm-up period before the actual study started, in which $\mathrm{E}$ engaged with the infant and elicited social behaviours like looking or smiling, and showing or giving objects. A between-subjects design was used. Each infant was randomly assigned to one of four conditions. In each condition there were 8 trials. Between trials infants were engaged in play with a bead-toy. On each trial, after E2 activated the assigned stimulus upon a signal by $\mathrm{E}$ (he touched his ear when he judged the child as attentive), $\mathrm{E}$ ignored the stimulus until the infant pointed to it, upon which he immediately began continuously reacting for so seconds. When an infant did not point on a given trial, E looked in a friendly way at the infant's face and uttered a few words or vocal expressions (e.g. 'uhmm, yeah, hmm'), to avoid a still-face situation. There was no set time limit for stimulus 


\begin{tabular}{|c|c|c|}
\hline \multirow[b]{2}{*}{ Reference } & \multicolumn{2}{|c|}{ Attitude } \\
\hline & $\begin{array}{c}\text { Shared } \\
\text { (positive affect) }\end{array}$ & $\begin{array}{c}\text { Not shared } \\
\text { (neutral affect) }\end{array}$ \\
\hline Shared (correct referent) & Joint Attention & Uninterested \\
\hline Not shared (incorrect referent) & Misunderstanding & No Sharing \\
\hline
\end{tabular}

exposure before the point, but if the infant did not point and uninterestedly looked away from the stimulus for more than 3 seconds, the stimulus was removed. On rare occasions (a total of 16 trials across 14 infants) a trial had to be excluded because infants did not point because they never looked at the stimulus due to fussiness or distraction. The following four conditions were administered (see Table 2 for a summary):

Foint Attention. There were no barriers. E alternated gaze between the stimulus and the infant's face twice, talking excitedly about the stimulus (but never naming it) and commenting on the fact that they were attending to the same thing. For example he said something like: 'Oh, that's nice! You are showing something neat to me.' When turning to the stimulus $\mathrm{E}$ slightly extended his arm, hand open, palm up in the direction of the stimulus (this was done to make it easier for infants to be sure of what he was looking at).

Misunderstanding. E alternated gaze between a barrier (the one which obstructed his line of sight to the stimulus) and the infant's face twice, talking excitedly about an incorrect referent (e.g. a small insignificant piece of paper attached to the barrier; again never naming it) and mistakenly commenting on the fact that they were attending to the same thing (as in Joint Attention). When turning to the barrier E extended his arm, hand open, palm up to touch the small piece of paper attached to the barrier.

Uninterested. There were no barriers. E reacted as in the Joint Attention condition except that he commented neutrally, not excitedly, about the event. He thus referred to the correct referent but was uninterested in it and commented in a non-positive way about it. He said something like: 'Hmm? Well, ... that's not really exciting ...'

No Sharing. E reacted as in the Misunderstanding condition, attending to the barrier, but he commented neutrally, as in the Uninterested condition. E thus misunderstood the infant's referent and uninterestedly referred to an insignificant object attached to the barrier.

\section{Coding and analyses}

Our main interest was in infants' pointing behaviour in response to E's reaction. Only trials with points were coded. Points were coded when the 
infant began extending the arm (either fully or slightly bent) and index finger or hand in the direction of the stimulus and lasted until the arm was withdrawn (either fully or more than half). A new point was coded when the arm was extended again after it had been withdrawn before. Points to non-test objects were not coded. We report results on the following main measures:

Trials with a point. Because not all infants pointed on every trial, and because some infants did not point on the first trial(s), this measure was computed as the number of trials in which infants pointed at least once as a proportion of the total number of trials (beginning with infants' first trial with a point).

Repetition of points. The number of trials in which infants repeated their pointing as a proportion of the number of trials in which infants pointed at least once (we also computed mean number of points per trial with pointing, as a secondary measure).

We also computed the duration of each point, the latency from the first look to the stimulus until the first point, and the latency from the end of the first point until the second point.

In addition, in order to further characterize infants' reactions, we also coded their looking and vocalizing in relation to their pointing. We expected that infants might look and vocalize more often to $\mathrm{E}$ when they were dissatisfied with E's reaction, in an attempt to communicate their dissatisfaction and to repair their communication if possible. Looks to the stimulus or to E's face were identified by movements of the infant's head and eyes. Communicative vocalizing was only coded when it was accompanied by looks to the stimulus or E; non-communicative sounds and fussing were not coded. Occurrences of vocalizing were coded as distinct when they were separated by a pause. We report results on the following additional measures: ( I) Looks to E's face: mean number and mean duration of looks to E's face per trial with a point; (2) Looks to the stimulus: mean number and mean duration of looks to the stimulus per trial with a point; and (3) Vocalizations: mean number and mean duration of vocalizations to $\mathrm{E}$ per trial with a point.

Coding of digital videotapes was done by E2, an assistant who was unaware of the hypotheses of the study, using the Interact computer programme (with the minimum unit of $\mathrm{I} / 25$ second $[=\mathrm{I}$ frame $]$ ) of the relevant behaviours. Except for vocalizing, which was coded in real-time, all behaviours were coded in different passes allowing for a frame by frame analysis. Inter-observer reliability was assessed in the same way by the first author, E, who coded a total of 8 randomly-selected infants ( 2 from each condition; not blind to condition or naïve to hypotheses). Cohen's Kappas were 0.96 for identification of each instance of pointing within a i s interval during a trial ( $n=87$ observations). Coding of duration of pointing was 
highly correlated, and the two coders did not differ from each other (means $=3.6 \mathrm{~s}$ and $3.4 \mathrm{~s} ;$ Pearson's $r=0.95, \quad p<0.00 \mathrm{I} ; t(69)=\mathrm{I} \cdot 44$, $p=0 \cdot 155)$. In those trials with pointing $(n=48)$ Cohen's Kappas for agreement on each second were 0.94 for looks to $\mathrm{E}, 0.90$ for looks to the stimulus, and 0.92 for vocalizing. For each of these measures we conducted one-way ANOVAs with condition as between-subjects factor and, if significant, planned comparisons (LSD) according to our hypotheses and post hoc comparisons (Dunnett, Tukey) where appropriate.

\section{RESULTS}

For some measures, that is, point duration, latency to point, and vocalizing, there were no statistically significant differences across conditions. For the secondary measure of point repetitions (mean number of points during trials with pointing) there were also no significant differences across conditions $(F(3,76)=\mathrm{I} \cdot 87, p=0 \cdot \mathrm{I} 4 \mathrm{I})$, although the pattern of results was similar to that of the main measure (mean proportion of trials in which infants repeated pointing; see Repetition of Points section below): means $=\mathbf{I} \cdot 4$ for Joint Attention, I· 6 for Misunderstanding, I· 3 for Uninterested, and I 4 for No Sharing. The lack of significant differences for the secondary measure mean number of points was most likely due to the fact that this measure is more susceptible than the main measure to individual differences, which cannot be well controlled in a between-subject design.

\section{Trials with a point}

A one-way ANOVA on the proportion of trials in which infants pointed at least once yielded a significant effect of condition, $F(3,76)=3 \cdot 30$, $p=0.025$. Following our first prediction, we tested the Joint Attention condition against each of the other conditions. Results confirmed our prediction that infants pointed on significantly more trials in the Joint Attention compared to the Misunderstanding, Uninterested, and No Sharing conditions (Dunnett, $p$ 's $=0.037,0.030$, and 0.006 , respectively; see Figure 2 for means and standard errors). Further, post hoc tests revealed that those three conditions were not significantly different from each other ('Tukey $p$ 's all $>0.490$ ). Sharing both attention and interest to the referent to which the infant pointed was thus the adult reaction that was most likely to elicit infant pointing on further trials.

Infants' dissatisfaction was particularly evident in the Uninterested condition. Whereas all infants in the Joint Attention condition pointed on at least one more trial after their first trial with a point, in the Uninterested condition 5 infants $(25 \%)$ never pointed again after their first trial with 


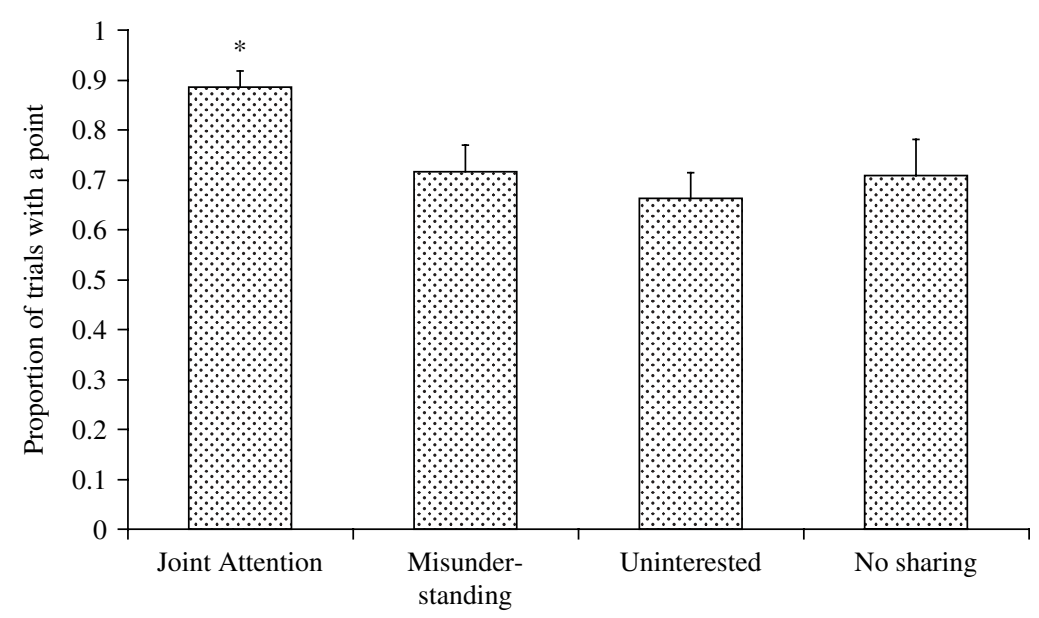

Fig. 2. Proportions of trials in which infants pointed at least once across the session.

* indicates a statistically significant difference compared to all other conditions.

a point (Fisher's exact chi-square comparison [2-tailed]: $p=0.047$ ). Comparisons between Joint Attention and Misunderstanding [ $\mathrm{I}$ infant never pointed again] and Joint Attention and No Sharing [2 infants never pointed again] were not significant.

\section{Repetition of points}

A one-way ANOVA on the mean proportion of trials in which infants repeated pointing yielded a significant effect of condition, $F(3,76)=2 \cdot 76$, $p=0.048$; see Figure 3 for means and standard errors. Following our second prediction, we compared the two positive comment conditions to test whether the mean proportion of trials in which infants repeated pointing was higher when E did not share the infant's referent (Misunderstanding condition) than when he did (Joint Attention condition). A planned comparison supported our prediction that when $\mathrm{E}$ expressed interest but referred incorrectly (Misunderstanding), infants repeated pointing within a trial on more trials than when $\mathrm{E}$ referred correctly (Joint Attention), $p=0 \cdot 055$. The two neutral comment conditions (Uninterested and No Sharing) did not differ significantly from each other.

Following our third prediction, we compared the two conditions in which $\mathrm{E}$ attended to the infants' referent to test whether infants repeated pointing within trials more when $\mathrm{E}$ did not share the infant's attitude (Uninterested condition) than when he did (Joint Attention condition). A planned comparison supported our prediction: infants did not repeat their pointing 


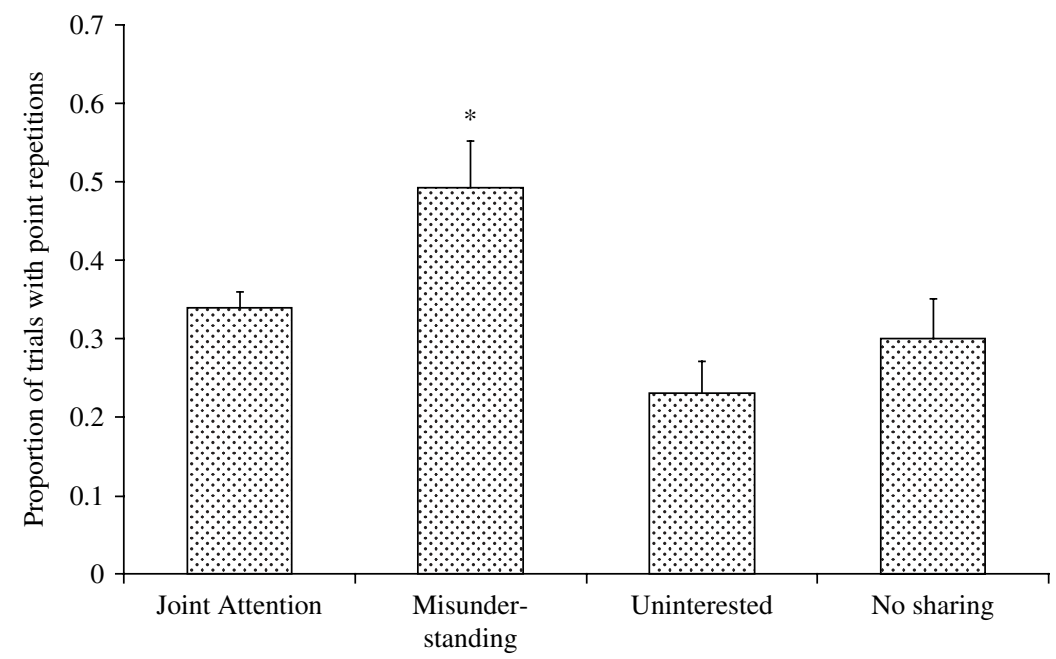

Fig. 3. Proportion of trials with at least one point in which infants repeated pointing to the same stimulus. * indicates a statistically significant difference compared to all other conditions.

within a trial on more trials when $\mathrm{E}$ emoted neutrally (Uninterested) than positively (Joint Attention), $p=0 \cdot 126$. Indeed, the number of infants who repeated pointing within a trial at least once across the session was significantly smaller in the Uninterested ( $\mathrm{I} / 20$ ) compared to the Joint Attention ( $18 / 20$ ) condition (Fisher's exact $\mathrm{c} 2=6 \cdot \mathrm{I}_{4}, p=0 \cdot 032$, two-tailed).

In order to see whether the point repetitions were selective to the Misunderstanding condition, we conducted a direct post hoc comparison between the two conditions in which infants' preferred response was violated but for different reasons, that is, when either the reference or the attitude component was violated (Misunderstanding and Uninterested conditions, respectively). In the Misunderstanding condition infants repeated pointing within a trial on significantly more trials than in the Uninterested condition (Tukey's $p=0.034,2$-tailed). Similarly, there was a tendency that more infants repeated pointing within a trial at least once across the session in the Misunderstanding than in the Uninterested condition (respectively, I $7 / 20$ and I I $/ 20$, Fisher's exact $\mathrm{c} 2=4 \cdot \mathrm{I} 8, p=0.082$, 2-tailed). Thus, infants differentially reacted to the different reasons behind the failure to share attention and interest.

Finally, to further analyze the difference between point repetitions in the Misunderstanding versus the Joint Attention condition we looked more closely at these repetitions in order to try to determine what infants were attempting to do with their repeated points. We therefore conducted a new 


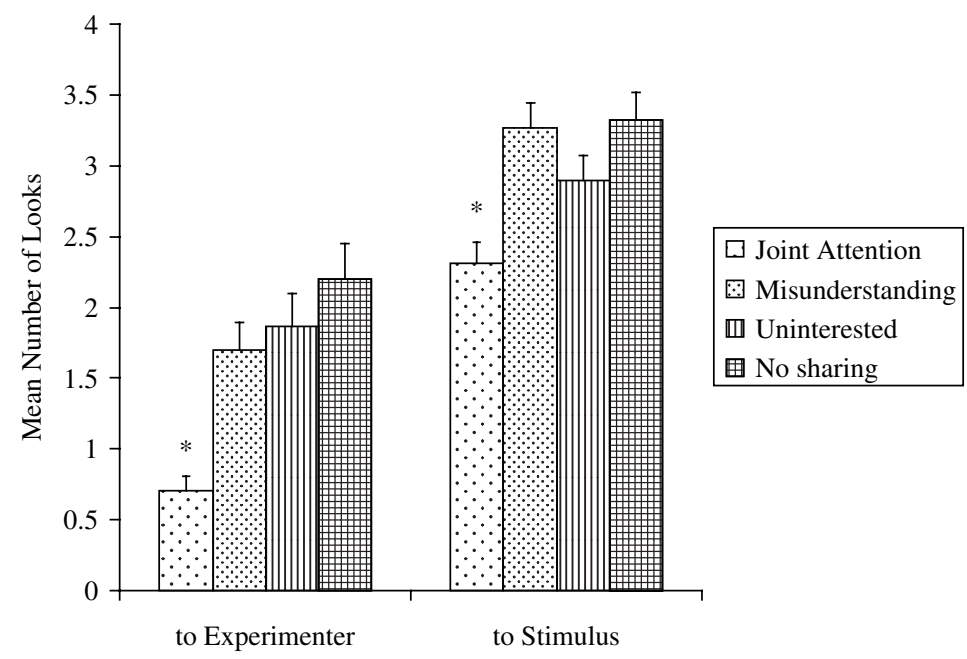

Fig. 4. Mean number of looks to $\mathrm{E}$ and to the stimulus during a trial with pointing.

* indicates a statistically significant difference compared to all other conditions.

set of analyses (independent t-tests, two-tailed) with the infants who repeated pointing within a trial in the Misunderstanding and Joint Attention conditions ( 8 and 17 infants, respectively), and focused only on those trials in which infants repeated pointing. We were interested in two behaviours on those trials with repeated points: looks to E's face and vocalizing. During the period between a point and a point repetition within a trial, infants looked significantly more often to $\mathrm{E}$ in the Misunderstanding $($ mean $=\mathrm{I} \cdot 49)$ than in the Joint Attention (mean $=0.49)$ condition, $t(33)=$ $2 \cdot 25, p=0.0$ I 6 (two-tailed), perhaps attempting to determine the problem or to call E's attention to their communicative intention. In addition, infants vocalized more often during their second point in the Misunderstanding $($ mean $=0.82)$ than in the Joint Attention (mean $=0.57)$ condition, $t(33)=$ I.7I, $p=0.050$ (two-tailed), perhaps attempting to augment their misunderstood signal.

\section{Looking behaviour}

Figures 4 and 5 display infants' looking pattern to E's face and to the stimulus in trials with a point. For looks to E's face, one-way ANOVAs on both the mean number and the mean duration of looks revealed significant effects of condition, $F(3,76)=$ I०.० I, $p<0.00 \mathrm{I}$ and $F(3,76)=$ I 2.00 , $p<0.00 \mathrm{I}$, respectively. Infants looked to E's face significantly more often and longer in all other conditions than in Joint Attention (Dunnett, all $p$ 's $<0.02$ ), presumably because in all other conditions $\mathrm{E}$ did not react as 


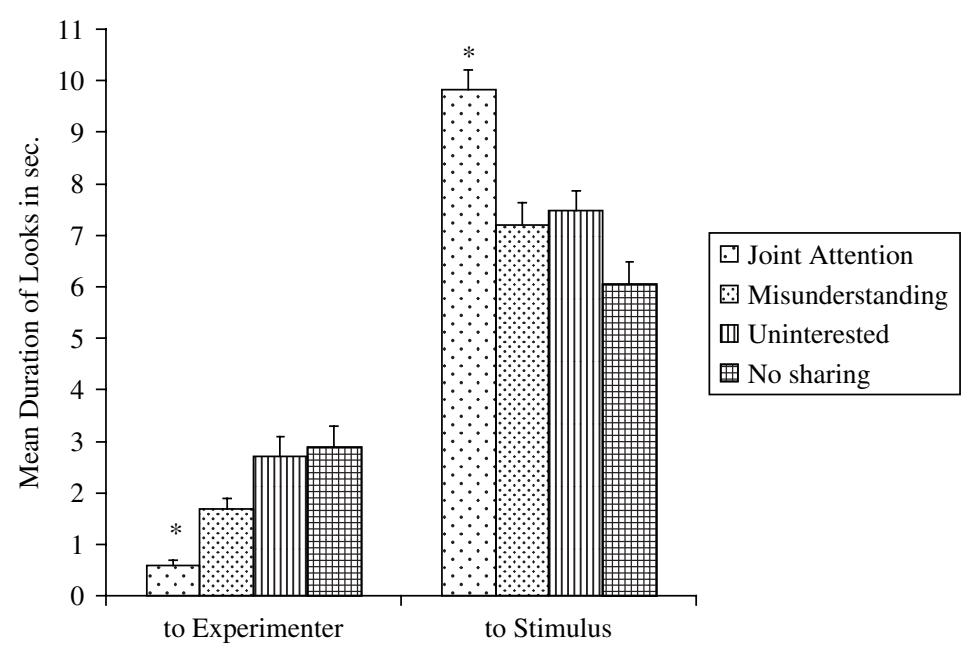

Fig. 5. Mean duration of looks to $\mathrm{E}$ and to the stimulus during a trial with pointing. * indicates a statistically significant difference compared to all other conditions.

they expected. For looks to the stimulus, one-way ANOVAs on the mean number and mean duration of looks revealed significant effects of condition, $F(3,79)=7.26, \quad p<0.00 \mathrm{I}$ and $F(3,79)=\mathrm{I}_{5} .67, \quad p<0.00 \mathrm{I}$, respectively. Infants looked less frequently but longer at the stimulus in the Joint Attention compared to all other conditions (Dunnett, all $p$ 's $<0.025$ ). Thus, overall, in the Joint Attention condition infants looked mainly at the stimulus, whereas in all other conditions they looked more often to E.

\section{ISCUSSION}

Together, the findings of this study support the sharing hypothesis. Infants pointed on significantly more trials when $\mathrm{E}$ reacted to their points by both identifying the correct referent and matching infants' positive attitude to it (Joint Attention condition) than when E matched their attitude but to the wrong referent (Misunderstanding condition) or matched their referent but showed a lack of interest in it (Uninterested condition) or both misidentified the referent and showed an uninterested, neutral attitude (No Sharing condition). In addition, infants looked more often and longer at E's face in these latter three mismatching conditions, presumably because they expected him to react differently. Thus, in pointing declaratively for others, twelve-month-old infants both want and expect the adult to share both their referent and their attitude toward it.

With regard to the reference component, results support the view that infants point to direct a person's attention specifically to what they are 
pointing at. Instead of merely wanting the adult to behaviourally turn in the general direction of the referent or to emote positively about anything, it was important to infants that the adult referred to the same event they were pointing at. When $\mathrm{E}$ did not do this and repeatedly misunderstood infants' referents, infants gave up and pointed less for $\mathrm{E}$ across trials. Furthermore, within trials, as predicted, infants repeated pointing in the Misunderstanding condition on more trials than in the Joint Attention condition, in an apparent attempt to redirect E's attention. This interpretation was supported by the fact that the point repetitions were accompanied by more behaviours such as increased looks to E's face and more vocalizing in the Misunderstanding than in the Joint Attention condition.

With regard to the attitude component, results also supported our interpretation that infants point to express and share interest about a referent that is jointly attended to. Infants were not satisfied and pointed in significantly fewer trials when $\mathrm{E}$ attended to the referent but did not share their interest, instead commenting neutrally (Uninterested condition). A quarter of the infants in that condition actually stopped pointing altogether after $\mathrm{E}$ responded to their first point in that way. Infants were thus not satisfied with just any type of comment from E; they specifically preferred a positive over a neutral one. The possibility that infants might point in this situation to request information (in a kind of social referencing) was not supported, as then one would have expected infants to point across trials independently of the type of information $\mathrm{E}$ conveyed. Further, results also did not support the hypothesis that infants simply point to obtain positive emotion or attention from adults. Under that hypothesis, when E referred to infants' referent infants should have repeated pointing persistently within a trial more often when $\mathrm{E}$ emoted neutrally (Uninterested condition) than positively (Joint Attention condition). This was not the case. Infants did not repeat pointing (like they do when unsuccessfully requesting an object) when $\mathrm{E}$ did not react as they wanted. In fact, about half of the infants in the Uninterested condition never repeated pointing on any trial. In addition, a comparison between the two conditions in which infants' preferred response was violated but for different reasons (Misunderstanding and Uninterested conditions) revealed that infants selectively repeated their pointing within a trial significantly more when $\mathrm{E}$ misunderstood the referent than when he was simply not interested in it. Thus, infants did not persist in or repair their message in the Uninterested condition like they did in the Misunderstanding condition, although their attempt to establish shared attention and interest failed here too. Instead, when $\mathrm{E}$ expressed his disinterest infants pointed less over trials and repeated pointing within trials less, presumably because they understood that $\mathrm{E}$ did not share their attitude towards the referent. 
Infants thus pointed in this context to align others' attention with their referent and share interest in it. However, in the No Sharing condition when E referred to an incorrect object and gave a neutral comment infants did not redirect E's attention. One interpretation of this response is that E's neutral comment simply put infants off, irrespective of whether he did or did not attend to the referent. It is possible that the neutrally emoted comment already indicated to infants that $\mathrm{E}$ did not want to share interest, even if he actually had not seen the referent.

\section{FOLLOW-UP EXPERIMENT}

The results on point repetitions in the Misunderstanding condition of the main experiment were in the predicted direction, but did not reach conventional significance levels. Two methodological factors may have contributed to this. First, the barriers and the fact that infants never saw $\mathrm{E}$ referring correctly in those conditions might have given infants the impression that $\mathrm{E}$ was not willing or able to refer to the event anyway, and so they did not repeat pointing on many trials. Second, we sometimes observed repeated pointing towards the location of the stimulus after the end of a trial, suggesting that the trial duration might have been not long enough to generate point repetitions. We thus attempted to improve these two aspects of the study in a follow-up experiment. In this experiment, therefore, we re-ran the two key conditions Joint Attention and Misunderstanding with some changes according to these two methodological factors. First, we used a within-subjects design, alternating conditions across trials, to ensure that infants would not consider $\mathrm{E}$ as generally unable or unwilling to attend to a referent. We also made the adult's misunderstanding more salient by using barriers in the Joint Attention condition too, such that $\mathrm{E}$ could demonstrate that he could peek around them. Second, to avoid the possibility that some point repetitions were initiated only after the end of a trial, we prolonged the trial duration.

\section{METHOD}

\section{Participants}

Twenty infants were recruited as in the main experiment. Two infants were excluded because of fussiness and one infant never pointed. Three infants pointed only in one of the two conditions. The final sample thus included 7 boys and 7 girls (mean age: I; O. I 9; range: I;0.7-I; I.5).

\section{Materials and set-up}

Materials were for most part the same as in the main experiment. The four windows left and right close to the infant's midline were again used for four 
different hand-puppets. Two windows left and right to the infant's midline were added, one for the wooden bird and one for another hand puppet which replaced the fan. Two objects (the lights) remained in front of the screen. Only the two barriers close to E were used. They differed in their length such that it was possible for $\mathrm{E}$ to peek around the shorter barrier when he turned his head and leaned forward.

\section{Procedure}

The general procedure was similar to that of the main experiment. Two conditions (Joint Attention, Misunderstanding; see main experiment) were administered to each infant, with the order of conditions alternating every two trials (irrespective of whether the infant pointed or not) and the first condition being counterbalanced across infants. Stimuli were always presented in the same order. In the Joint Attention condition E reacted to the four stimuli on the side of the shorter barrier, leaning forward to peek around it following infants' pointing, and in the Misunderstanding condition he reacted directly to the longer barrier, leaning forward to inspect an insignificant piece of paper attached to the barrier. E reacted continuously to infants' pointing for I 5 seconds, 5 seconds longer than in the main experiment. The positions of the barriers were counterbalanced for side, as was the position of the mother, who sat approximately $\mathrm{I} \cdot 5 \mathrm{~m}$ away and slightly behind the infant.

\section{Coding}

We were primarily interested in infants' point repetitions. In this experiment, because we used a within-subjects design with fewer trials per condition and conditions that alternated every two trials irrespective of infants' pointing (and so infants did not have the same opportunity as in the main experiment to develop an expectation about how $\mathrm{E}$ would react to their points), it was more appropriate to use as our main measure the mean number of points per trials with pointing (instead of mean proportion of trials with repeated points, which we report briefly as a secondary measure). For the same reasons, we report no analyses on the measure Trials with a Point. In addition, we also coded infants' looking behaviour and vocalizing. Coding was done as in the main experiment by the same student assistant. Reliability was again assessed by the first author, E, who coded three infants of the final sample. Cohen's Kappas were excellent: for identification of each instance of pointing within a $\mathrm{I} s$ interval during a trial $(n=3 \mathrm{I}$ observations), kappa $=\mathbf{I} \cdot 0$, and for trials with pointing $(n=9)$ kappas for vocalizing, looks to E's face, and looks to the stimulus on a I-s base were $\mathrm{I} \cdot 0,0 \cdot 84$, and $0 \cdot 90$, respectively. 
RESULTS

Infants pointed on average on $60 \%$ of the total trials. The condition in which infants pointed first had no significant effect on their pointing in subsequent trials. For the secondary measure of pointing, mean proportion of trials in which infants repeated pointing, there was a similar pattern of results to that in the main experiment for the Misunderstanding and Joint Attention conditions (means $=0.44$ and 0.30 , respectively) but this difference did not reach statistical significance $\left(t\left(\mathrm{I}_{3}\right)=\mathrm{I} \cdot 33, p=0 \cdot \mathrm{I}_{3}\right.$, I-tailed), most likely because power was reduced compared to the main experiment due to the within-subjects design with fewer trials per condition. For vocalizing there were no statistically significant differences between conditions.

\section{Repetition of points}

A one-tailed paired comparison on the mean number of points in trials with a point confirmed our prediction that infants pointed significantly more often within a trial in the Misunderstanding $($ mean $=\mathrm{r} \cdot 6)$ than in the Joint Attention condition (mean $\left.=\mathrm{I}_{3}\right), t\left(\mathrm{I}_{3}\right)=2 \cdot 29, p=0 \cdot 020$. No further analyses on repeated point characteristics, such as those done in the main experiment, were conducted because too few infants repeated pointing within a trial in вотн conditions (necessary for repeated-measure analyses).

\section{Looking behaviour}

Infants' number and duration of looks to E's face were not significantly different between conditions. However, there was a significant effect of order such that infants looked more often to E's face when he had reacted to their first point with Misunderstanding than with Joint Attention, $F(\mathrm{I}, \mathrm{I} 2)=5.84, p=0.033 ;$ means $=2.2$ and $\mathrm{I} \cdot 2$, respectively. Like in the main experiment, infants looked significantly longer to the stimulus in the Joint Attention than in the Misunderstanding condition, $F(\mathrm{I}, \mathrm{I} 2)=\mathrm{I} 9 \cdot 2$, $p=0.00 \mathrm{I} ;$ means $=\mathrm{I} \mathrm{s}$ and $7 \mathrm{~s}$, respectively. The number of looks to the stimulus did not differ significantly between conditions.

\section{DISCUSSION}

Findings on infants' point repetitions in this experiment are consistent with those of the main experiment. Infants pointed significantly more often to the same referent when $\mathrm{E}$ referred to an incorrect referent than when he referred to the correct one, apparently to repair the failed communication and redirect E's attention to the correct referent. In addition, infants looked more to $\mathrm{E}$ when he first reacted to their pointing in the Misunderstanding condition, presumably - and in line with the main experiment-because 
they found his reaction odd. When $\mathrm{E}$ reacted in Joint Attention infants looked longer to the stimulus - again in line with the main experiment suggesting that they were less surprised or distracted by E's reaction when he referred to the correct referent and so they sustained attention to the referent more. Other measures did not produce statistically significant differences between conditions, most likely because power was lower than in the main experiment (infants had only four trials per condition and the sample size was much smaller). Taken together, both experiments show that twelve-month-olds point referentially to direct a person's attentional state towards the particular external event at which they point.

\section{GENERAL DISCUSSION}

Overall, the current findings support the hypothesis that infant pointing at twelve months is already a full communicative act involving both reference and attitudes towards that referent, while excluding several alternative hypotheses. This suggests both a social-cognitive understanding of other persons as agents with attentional states and attitudes, and a motive to share experiences with others.

Communicative pointing reveals a social-cognitive understanding of attention and attitudes in the sense that an indication is about something and that it expresses psychological relations between interlocutors and referents. To understand reference, one has to understand others' attentional relation to the environment. It requires an understanding of others as intentionally perceiving and singling out entities in the environment, not just behaviourally orienting in some direction. In line with recent results on gaze following (Brooks \& Meltzoff, 2002; Deák et al., 2000) and social referencing (Moses et al., 200I), this study shows that twelve-month-olds understand what other people attend and refer to. In addition, it shows that infants actively direct others' attention, and even redirect others' attention when they are mistakenly referring to an incorrect referent. This 'message repair', which was selective to the Misunderstanding condition, can be interpreted as the infant helping an interested partner in achieving the communicative goal of shared reference (see also Golinkoff, r 986 ).

To understand a person's attitude one has to understand that person's psychological relations towards the referent. Moses et al. (200I) have shown that twelve-month-olds, when socially referencing, link an adult's comment selectively to the object the adult is attending to. Recently, it has been proposed that infants relate these comments only directly to the object, as its valence, but not to the sender as expressing his or her psychological relation to the object (Egyed, Kiraly \& Gergely, 2004). But the current study shows that infant pointing in this context does not involve requesting valence information about an object. Instead it is to share psychological 
relations with others about a referent. Results showed that when an adult reacted uninterestedly, infants ceased pointing for him. Our interpretation is that infants understood E's attitude about the referent as different from their own, that is, as not wanting to share their interest in the referent. Perhaps this is because in this context, infants already had their own pre-determined attitude about the object (i.e. one of positive interest) whereas in social referencing situations the object is designed to be ambiguous to infants. Infant pointing thus enables social learning not only about the external environment (Csibra \& Gergely, 2005) but also about the psychological relations between other persons and the environment, which are more subject to change than stable object properties.

Infants point for various motives (Liszkowski, in press). One such motive is to REQUEST HELP from adults in getting something done. Another motive is to PROVIDE HELP to adults by informing them of things they want or need to know ('informative' pointing; Liszkowski, Carpenter, Striano \& Tomasello, 2006). Here (see also Liszkowski et al., 2004), we have investigated infant 'proto-declarative' pointing and provided empirical evidence for an underlying motive of SHARING ATTENTION AND INTEREST. This motive is distinct because it is neither purely to inform ('direct attention'; Baron-Cohen, I99I) nor purely to request ('obtain attention'; Moore \& D'Entremont, 200I). Instead, it is an offer - an 'invitation' (see also Bruner, I983) - to jointly engage with a communicative partner and experience something together.

The current study thus demonstrates that preverbal infants' intentional communication already resembles adult language and conversation in many important ways. Infant declarative pointing at twelve months, before language has emerged, already is a full communicative act embodying two main components of adult linguistic speech acts: reference and attitude about referents (see Searle, I969). In addition, it already has some of the collaborative structure of adult conversation (see Clark, I 996), as seen when infants repair their failed communications, actively working to align their communicative partner's focus of attention with their own (Golinkoff, I986). This study thus provides further support for the social-pragmatic account of language development (see, e.g. Tomasello, 2003), demonstrating already in infants' preverbal communication the presence of the same underlying abilities and motivations upon which language is built.

\section{REFERENCES}

Baldwin, D. A. \& Moses, L. J. (1996). The ontogeny of social information gathering. Child Development 67, 1915-39.

Baron-Cohen, S. (I99I). Precursors to a theory of mind: understanding attention in others. In A. Whiten (ed.), Natural theories of mind: evolution, development and simulation of everyday mindreading. Oxford: Blackwell. 
Bates, E., Camaioni, L. \& Volterra, V. (1975). The acquisition of performatives prior to speech. Merrill-Palmer Quarterly 21, 205-26.

Brooks, R. \& Meltzoff, A. N. (2002). The importance of eyes: how infants interpret adult looking behaviour. Developmental Psychology 38, 958-66.

Bruner, J. (I983). Child's talk. New York; London: Norton.

Camaioni, L. (I993). The development of intentional communication: A re-analysis. In J. Nadel \& L. Camaioni (eds), New perspectives in early communicative development, 82-96. London: Routledge.

Clark, H. (1996). Uses of language. Cambridge: CUP.

Csibra, G. \& Gergely, G. (2005). Social learning and social cognition: the case for pedagogy. In M. H. Johnson \& Y. Munakata (eds), Processes of Change in Brain and Cognitive Development. Attention and Performance XXI. Oxford: Oxford University Press.

Deák, G. O., Flom, R. A. \& Pick, A. D. (2000). Effects of gesture and target on I2- and I 8-month-olds' joint visual attention to objects in front of or behind them. Developmental Psychology 36, 5 I I-23.

Egyed, K., Kiraly, I. \& Gergely, G. (2004). Object-centered versus agent-centered interpretations of attitude expressions. Poster presented at the International Conference on Infant Studies, Chicago.

Golinkoff, R. (I 986). 'I beg your pardon?': The preverbal negotiation of failed messages. Fournal of Child Language $\mathbf{1 3}, 455-76$.

Golinkoff, R. (I993). When is communication a 'meeting of minds'? Fournal of Child Language 20, 199-207.

Liszkowski, U. (in press). Infant pointing at twelve months: Communicative goals, motives and social cognitive abilities. In N. Enfield \& S. Levinson (eds), The roots of human sociality: culture, cognition, and interaction. Oxford: Berg.

Liszkowski, U., Carpenter, M., Henning, A., Striano, T. \& Tomasello, M. (2004). Twelvemonth-olds point to share attention and interest. Developmental Science 7, 297-307.

Liszkowski, U., Carpenter, M., Striano, T. \& Tomasello, M. (2006). Twelve- and I 8-month-olds point to provide information. Fournal of Cognition and Development 7 , I $73-87$.

Moore, C. \& D'Entremont, B. (200I). Developmental changes in pointing as a function of attentional focus. Fournal of Cognition and Development 2, I09-1 29.

Moses, L. J., Baldwin, D. A., Rosicky, J. G. \& Tidball, G. (200I). Evidence for referential understanding in the emotions domain at twelve and eighteen months. Child Development 72, 7 I $8-35$.

Searle, J. R. (1969). Speech acts. Cambridge: CUP.

Tomasello, M. (2003). Constructing a language: a usage-based theory of language acquisition. Harvard University Press. 